\title{
Après la quête de la survie, les traitements du cancer peuvent-ils ramener le désir ?
}

\author{
Beyond the Survival Quest Are Cancer Treatments Able \\ to Bring Back Desire?
}

\author{
M.-F. Bacqué \\ (C) Lavoisier SAS 2016
}

L'évolution des représentations de la sexualité des patients atteints de cancer mérite une nouvelle mise au point. Après un silence absolu des patients devant les préjugés séculaires qui longtemps attribuèrent une sexogenèse au cancer, deux tentatives de normalisation de la sexualité résultent des années 2000 : une normalisation fonctionnelle et une normalisation sociale.

Et d'abord, le désir et, partant, la sexualité du patient existaient-ils avant le cancer ? Volontaires quant à l'idée de ne pas frustrer encore plus les patients, les professionnels du cancer ont postulé, un peu vite, qu'il fallait leur redonner leurs capacités antérieures. On découvrait alors que, pour certains, cette sexualité, ce Graal des années peace and love n'avait jamais été atteint. Ce fut la première déconvenue des soignants, médecins, infirmières, psy, qui auraient tant aimé agir " du côté de la vie ». En réalité, les stéréotypes des soignants se trouvaient des deux côtés : redonner la sexualité présumée était conçu comme un traitement implicite du cancer ou comme un indicateur de la régression de la maladie, perdre sa sexualité relevait alors forcément de la mort. Or, pour certains, assumer la fin de la sexualité d'organe ne signifiait pas renoncer à la sexualité globale.

Mais rien n'est plus compliqué que la sexualité humaine...

Premier point, la sexualité n'est pas qu'une affaire génitale. La sexualité est globale chez la femme, et, n'en déplaise aux pornographes, elle l'est aussi chez l'homme.

\section{La médecine ne peut se contenter d'une normalisation fonctionnelle de la sexualité}

Nous entendons déjà la protestation des oncologues : «Encore ! Il faut maintenant prendre en charge la sexualité !»

M.-F. Bacqué $(\bowtie)$

Rédactrice en chef de Psycho-Oncologie,

EA 3071, université de Strasbourg, France

e-mail : mfbacque@club-internet.fr
Les biopouvoirs de la médecine ont été critiqués depuis Michel Foucault, et les médecins préfèrent aujourd'hui se retrancher derrière leur clinique, celle, déjà suffisamment ardue, du diagnostic et des programmes thérapeutiques du cancer. Si la sexualité peut être abordée par le biais d'une ou deux questions qui passent encore, écouter le patient s'exprimer sur le sujet entraine des risques : ne va-t-on pas entrer dans une intimité trop... personnelle ? En quoi la sexualité va-t-elle améliorer la qualité de vie ? Ou le pronostic?

En cette période de pénurie des oncologues, ceux-ci se contenteraient bien d'une seule question et passeraient la main aux spécialistes. La question est déjà difficile : « Comment ça se passe au niveau des... Au niveau des relations sexuelles?» ou encore, plus risqué : « Avec votre conjoint, ça va ?» On l'aura compris, la plupart des oncologues préféreraient attendre la plainte des patients. Hélas, celle-ci dépend à son tour de nombreuses configurations sociales : des éléments facilitateurs comme le fait que l'oncologue ait le même sexe que son patient, ou encore qu'une relation de confiance se soit tissée entre eux, que le patient soit suffisamment à l'aise avec la sexualité pour l'aborder avec son médecin, que le médecin soit suffisamment à l'aise avec la sexualité pour l'aborder avec son patient... L'observation la plus courante aujourd'hui est celle du retranchement du médecin dans une sexualité fonctionnelle : « et du côté de... Comment ça marche ?»

Or, cette sexualité liée à la mécanique du corps concerne préférentiellement les hommes et, après un cancer de la prostate, dévoile deux types de problèmes ; l'érection et la continence urinaire. Chez les femmes, le cancer du sein atteindrait l'organe cible d'une sexualité stéréotypée avec un sein cicatriciel, absent ou reconstruit. Le questionnement sur la sexualité s'imposerait de lui-même, il toucherait cette partie du corps et permettrait de déplacer l'angoisse de mort sur l'atteinte du triple symbole de la beauté, de la féminité et de la maternité. Mais pour les hommes, comme pour les femmes, la centration sur la fonction sexuelle oublie la personne... 


\section{La sexualité a pourtant une dimension globale}

L'approche partielle de la sexualité a ses limites, bien repérées maintenant par les médecins et les soignants et mises en évidence par l'approche psychologique de la sexualité. Si S. Freud a permis de franchir le tabou, il n'a pas pour autant débarrassé la sexualité de ses stéréotypes. Les travaux de W.H. Masters et V.E. Johnson y ont sans doute plus fortement contribué, mais toujours sous un angle mécanique, renonçant à prendre en compte les aspects psychosociaux de la sexualité. La sexualité est finalement plus abordée dans la littérature depuis le début $\mathrm{du} \mathrm{xx}^{\mathrm{e}}$ siècle, alors que l'amour a toujours été célébré depuis les débuts de l'écriture.

L'amour «parle aux affects », tandis que la sexualité s'adresserait au corps. L'amour serait donc une caractéristique culturelle humaine, alors que l'acte sexuel nous rapprocherait de l'animalité. Finalement, deux « sexualités » émergent de ces constats, une sexualité "d'organe », sexualité fonctionnelle, simple à aborder puisqu'elle relèverait de l'avoir, « en avoir ou pas, là est la question » et une sexualité psychique, donc globale, qui relèverait de l'être et poserait la question existentielle d'Hamlet cette fois.

Que nous rapporte l'approche approfondie de l'histoire de ces femmes et de ces hommes, lorsqu'ils veulent bien parler de ce qui, avec le cancer, les mine ?

Pour ce qui est de l'extérieur, l'opération chirurgicale visible est souvent synonyme de brutalité et de radicalité. Elle entache parfois la beauté, la symétrie, la régularité. Avec ces altérations est alors suspendue l'expérience subjective de la continuité de soi, de ce qui fait son identité profonde. Les différentes chimiothérapies provoquent souvent amaigrissement ou obésité morbides, elles modifient la couleur de la peau, donnent un teint parfois anémié, cireux, contradictoire avec le besoin inconscient et relevant de l'éthologie humaine de partager son potentiel sexuel (de reproduction ?) avec une personne visiblement en bonne santé. Pour les cancers en général, la fatigue liée à la maladie et à ses tentatives thérapeutiques (chimio-, radio- et immunothérapie) se traduit par une incapacité à être actif pendant l'amour. La sexualité humaine, c'est aussi cela : séduire avec son corps, sa manière de bouger, son activité et sa passivité, sa capacité à jouer ou à prendre au sérieux le désir de l'autre, ses soupirs, ses mots doux ou crus, son alternance de petites frustrations et de grands élans communs...

Hélas, une certaine violence de l'annonce de la maladie et des traitements éveille ou réveille des traumatismes passés. Et puis n'oublions pas les particularités de certains cancers : ceux de la face qui brouillent l'identité, ceux des voies aériennes qui bloquent la voix ou transforment son timbre en un son insupportable. Pensons aussi à tous les cancers abdominaux qui augmentent la sensibilité, empêchant d'avoir un poids sur soi. Les questions d'odeurs sont parfois aussi insurmontables que la vision de l'aimé déformé ; odeur médicamenteuse ou odeur excrémentielle, autant de difficultés avec les cancers ORL, colorectaux et de la sphère anale. Le malade, sa compagne ou son compagnon peuvent-ils dépasser l'empreinte corporelle du cancer ?

\section{On est donc loin du stéréotype de genre : les hommes malades du cancer rencontreraient des problèmes mécaniques, les femmes auraient des difficultés émotionnelles}

Les soignants partagent avec la majorité les mêmes stéréotypes sur la sexualité. L'enquête menée par Giami et al. est claire [1]. Dans les années 1970 prévalaient encore deux préjugés distincts sur l'étiologie du cancer chez les hommes et les femmes. L'excès de sexualité chez les femmes serait à l'origine de plus de cancers du col de l'utérus. Au contraire, le développement sexuel tardif, la répression de la sexualité à l'adolescence ou encore la cessation prématurée de la sexualité conduiraient au cancer de la prostate. La sexualité, l'hypersexualité s'avéraient dangereuses pour la femme, l'absence de sexualité l'était pour l'homme.

L'influence de médecins se réclamant de la psychanalyse (on ne soulignera jamais assez combien S. Freud refusait de soutenir ces thèses), comme Groddeck [2] et Reich [3], s'est diffusée jusqu'à aujourd'hui, puisque si la psychogenèse du cancer est rejetée le plus souvent en persistent des bribes qui conduisent les infirmières à dire que « le moral fait toute la différence, c'est $50 \%$ de la guérison » [4]. Ce sont souvent les infirmières qui recueillent les questions des patients. Or, ce sont les moins bien formées actuellement. Leur formation « cognitive » est en cause, mais apprendre à connaître les dysfonctions sexuelles n'est pas un obstacle. Leurs réticences à aborder la sexualité concernent surtout les patients différents (d'elles) : ceux de sexe opposé, d'orientation sexuelle différente, d'âge éloigné, d'appartenance ethnique et culturelle autre. Enfin, elles se méfient des célibataires. Ce constat de É. Huyghe et al. met bien en évidence la même problématique qu'avec les médecins : on peut proposer autant de formations consistant à ingérer des savoirs. Tant que l'on ne travaille pas en profondeur la relation soignant-soigné, les stéréotypes persistent [5]. Travailler en groupe Balint à l'intégration des éléments inconscients toujours présents dans les transactions thérapeutiques permet d'aller beaucoup plus loin que dans la simple éducation thérapeutique : questionner son patient est une chose, se questionner soi en est une autre, le second questionnement devrait toujours éthiquement précéder le premier [6]. C'est alors que les pensées préformatées disparaîtront et que les patients, bénéficiant d'une véritable écoute, pourront poser leurs véritables questions. 


\section{Émergence de l'onco-sexologie}

Véritablement née en 2006 d'après Eliane Marx, l'oncosexologie montre une évolution majeure dès 2011 : «Faire que l'on puisse parler de sexualité aux oncologues et de cancer aux sexologues » [7]. Certes, il y a du changement. Cependant, dès que la sexualité a été prise en compte, «c'est la santé sexuelle qui a été promue », selon Giami [8]. Il est évidemment plus facile pour un médecin d'aborder la question sexuelle par le biais de la santé. Mais ici, la peur de partager l'intimité du patient s'est munie d'une défense intéressante : il s'agissait de désérotiser la sexualité, de la médicaliser afin de lui ôter cette part subjective risquant de la faire glisser au cœur même de la relation médecin-soignantmalade. Des onco-sexologues ont bien vu le risque de cette remédicalisation de la sexualité. Heureusement, psychologues et sexologues le manifestent nettement, il faut d'abord être à l'écoute du patient. Cette écoute et cette disponibilité peuvent être très étayantes pour eux. Cependant, pour que cela se sache, une information devrait être divulguée sur les sites comme dans les services d'oncologie. Des plaquettes ou des affiches " la sexualité, parlons-en » ou encore « des questions difficiles ? et proposant de s'adresser à un soignant particulièrement à l'écoute et formé en conséquence pourraient aider les patients.

Des systèmes de pairs-experts peuvent aussi favoriser la parole. L'expérience d'É. Garand-Castello le montre nettement : au sein d'un groupe masculin, coanimé par un psychologue et un ex-patient, les patients atteints d'un cancer de la prostate s'expriment beaucoup plus franchement. Ils ne craignent plus de partager leurs inquiétudes, leurs peurs et certaines humiliations ou sujets de honte. Le contraste est grand avec les méthodes projectives qui montrent, lors d'un examen psychologique, les défenses massives de patients 8 à 12 semaines après leur prostatectomie. L'attaque du narcissisme semble alors à son acmé, les patients relèvent tout manque de la planche de Rorschach et s'en défendent par de nombreux traits hypomaniaques. À ce stade, ils évitent la confrontation à la castration et au manque, plutôt que de sublimer la réalité. Cette attitude (masculine ?) est aussi une manière de réagir contre l'attaque du moi. Elle ne devrait pas persister longtemps et mériterait d'être relativisée par une compréhension longitudinale.

Le choix des articles effectué par Eliane Marx témoigne de l'évolution d'une nouvelle spécialité, l'onco-sexologie.
Celle-ci peut, sans conteste, apporter de nouvelles connaissances techniques sur les moyens de maintenir sa sexualité après un cancer. Mais sur le plan personnel, nombreux sont les patients qui n'en sont pas là. Tout d'abord parce que les cancers atteignent majoritairement des personnes de plus de 60 ans et que de nombreuses générations de cet âge ou plus âgées dévalorisent la sexualité. Par ailleurs, la menace mortelle du cancer implique un réaménagement des priorités des patients et de leur couple, une nouvelle hiérarchie s'impose, celle de la survie. Enfin, si les patients et leur conjoint expriment des plaintes, les médecins et les soignants n'ont pu les recevoir vraiment qu'après s'être formés. Les formations ont permis deux changements : accepter de poser la question de l'évolution de la sexualité, replacer la sexualité dans une approche holistique de la personne sans la limiter à un dysfonctionnement d'organe. L'évidence est là : pour approcher la sexualité globale, il faut être à l'écoute du patient. Le principe de l'écoute clinique du patient repose sur l'attente bienveillante de l'expérience subjective du patient et sur le travail interne du thérapeute qui lui permettra de se défaire de ses préjugés pour favoriser la réflexion du patient vers sa réelle quête : celle de l'intégration du changement et du retour de l'autonomie psychique.

\section{Références}

1. Giami A, Moreau E, Moulin P (2015) Infirmières et sexualité : entre soins et relations. Éditions de l'EHESP, Rennes

2. Groddeck GW (1978) Conférences psychanalytiques à l'usage des malades, trois volumes. Champ Libre, Paris, pp 79 et 81 . Volume 1 : Conférences 1 à 40 (16 août 1916-23 mai 1917). Volume 2 : Conférences 41 à 80 (30 mai 1917-20 mars 1918). Volume 3 : Conférences 81 à 115 (27 mars 1918-2 avril 1919)

3. Reich W $(1936,1982)$ La révolution sexuelle. Christian Bourgois, Paris

4. Soum-Pouyalet F, Vega A, Cousson-Gélie F (2009) Le « moral» en cancérologie. Stratégies et discours des soignants dans l'accompagnement des malades. Psycho-Oncologie 3:161-7

5. Bacqué MF (2009) La charge symbolique du cancer. PsychoOncologie 3:4-6

6. Balint M, Balint E, Gosling R, Hildebrand P. (1966) A study of doctors. Tavistock Publication, London

7. Incrocci L (2007) Cancer et fonction sexuelle : parler de sexualité aux oncologues et de cancer aux sexologues. Sexologies 16:263-4

8. Giami A (2005) Santé sexuelle : la médicalisation de la sexualité et du bien-être. Comprendre 6:97-115 